\title{
Influence of the four-fermion interactions in a $(2+1) D$ massive electron system
}

\author{
Luis Fernández ${ }^{1, * *}$ Van Sérgio Alves $\oplus^{1, \dagger}$ M. Gomes $\oplus^{2, \ddagger}$ Leandro O. Nascimento $\oplus^{3, \S}$ and Francisco Peña $\oplus^{4, \|}$ \\ ${ }^{1}$ Faculdade de Física, Universidade Federal do Pará, 66075-110 Belém, Pará, Brazil \\ ${ }^{2}$ Instituto de Física, Universidade de São Paulo, Caixa Postal 66318, \\ 05315-970 São Paulo, São Paulo, Brazil \\ ${ }^{3}$ Faculdade de Ciências Naturais, Universidade Federal do Pará, C.P. 69900-000 Breves, Pará, Brazil \\ ${ }^{4}$ Departamento de Ciencias Físicas, Facultad de Ingeniería y Ciencias, Univesidad de La Frontera, \\ Avenida Francisco Salazar 01145, Casilla 54-D, Tетисо, Chile
}

(Received 14 March 2021; accepted 23 April 2021; published 19 May 2021)

\begin{abstract}
The description of the electromagnetic interaction in two-dimensional Dirac materials, such as graphene and transition-metal dichalcogenides, in which electrons move in the plane and interact via virtual photons in $3 \mathrm{D}$, leads naturally to the emergence of a projected theory, called pseudo-quantum electrodynamics (PQED), as an effective model suitable for describing electromagnetic interaction in these systems. In this work, we investigate the role of a complete set of four-fermion interactions in the renormalization group functions when we coupled it with the anisotropic version of massive PQED, where we take into account the fact that the Fermi velocity is not equal to the light velocity. We calculate the electron self-energy in the dominant order in the $1 / N$ expansion in the regime where $m^{2} \ll p^{2}$. We show that the Fermi velocity renormalization is insensitive to the presence of quartic fermionic interactions, whereas the renormalized mass may have two different asymptotic behaviors at the high-density limit, which means a high-energy scale.
\end{abstract}

DOI: 10.1103/PhysRevD.103.105016

\section{INTRODUCTION}

Four-fermion interactions have been extensively studied in the literature, both for understanding conceptual aspects of quantum field theory as well as for applications in condensed matter physics. In particular, the Thirring [1] and Nambu-Jona-Lasinio [2] models show a rich connection between the phenomenon of superconductivity and elementary particle physics. The latter has also been used for studying quantum chromodynamics at the low-energy limit $[3,4]$. Although four-fermion interactions are perturbatively non-renormalizable in a space-time $D>2$, in the sense of general power counting rules [5], they become renormalizable when we use the $1 / N$ expansion in $D=3$ [6]. Indeed, the incorporation of vacuum polarization effects provides a better behavior for the Green functions in the ultraviolet regime. Therefore, both the Gross-Neveu [7] and

\footnotetext{
*luis.aguilar@icen.ufpa.br

vansergi@ufpa.br

"mgomes@fma.if.usp.br

§lon@ufpa.br

"francisco.pena@ufrontera.cl
}

Published by the American Physical Society under the terms of the Creative Commons Attribution 4.0 International license. Further distribution of this work must maintain attribution to the author(s) and the published article's title, journal citation, and DOI. Funded by SCOAP.
Thirring [8] interactions may be renormalizable in $D=3$. Usually, in order to perform the $1 / N$ expansion, a HubbardStratonovich transformation [9] is used through the introduction of an auxiliary field, which has no dynamics at the tree level.

It is well known that the quasiparticle excitations in twodimensional materials at the honeycomb lattice (such as graphene [10], silicene [11], and transition metal dichalcogenides [12]) behave as Dirac-like fermions (either massless or massive). Hence, the four-fermion interactions also become relevant, as an attempt to obtain a more complete description of these systems, within a quantum-field-theory approach. Indeed, this more realistic description should take into account some of the microscopic interactions that, such as disorder or impurity, may emerge in these materials. Because the auxiliary fields obey the same properties as the random disorder or impurities interactions, as discussed in Refs. $[13,14]$, hence, we can relate these properties of the materials with the four-fermion interactions within the lowenergy limit. Furthermore, it is also very useful to consider the electromagnetic interactions in the plane, which may be effectively described by the pseudoquantum electrodynamics model [15].

In a previous work, we analyzed the effect of the electromagnetic interaction on the renormalization of the mass gap of electrons moving in a plane subject also to impurities simulated by a Gross-Neveu like self-interaction [16]. Without the four-fermion interaction, we derived 
results that are in excellent agreement with experimental measurements of the band gap for $\mathrm{WSe}_{2}$ [17] and $\mathrm{MoS}_{2}$ [18]. We found also that, although the presence of the Gross-Neveu like interaction does not alter the renormalization of the Fermi velocity [19], it provides an ultraviolet fixed point in terms of an effective fine-structure constant so that the renormalized mass has different behaviors below and above it.

In this paper, we extend the investigation presented in [16] by considering the generalized four-fermion interactions with $O(4)$ symmetry.

The remainder of this paper is organized as follow. In Sec. II, we present our model, notation, and perform the expansion $1 / N$ through the Hubbard-Stratonovich transformation, which allows us to define the Feynman rules. In Sec. III, we calculate the propagators of the gauge and auxiliary fields in the dominant order in $1 / N$ in the regime where $m^{2} \ll p^{2}$. In Sec. IV, we calculate the electron selfenergy due to electromagnetic and the four-fermion interactions, taking into account the effect of the polarization tensor obtained in the previous section. The derivation of the renormalization group functions and the effect of each four-fermion interaction on the renormalized mass are shown in Sec. V. In Sec. VI, we review our main results and conclusions. Some details about the derivation of the polarization tensor, due to the four-fermion interactions, are given in the Appendix.

\section{PSEUDOQUANTUM ELECTRODYNAMICS WITH FOUR-FERMIONS INTERACTION}

We consider the PQED model [15] with a complete set of independent four-fermion interactions in $(2+1) \mathrm{D}$ [20]. The Euclidean action reads

$$
\begin{aligned}
\mathcal{L}= & \frac{1}{2} \frac{F^{\mu \nu} F_{\mu \nu}}{\sqrt{-\square}}+\bar{\psi}_{a}\left(i \gamma^{\mu} D_{\mu}-m\right) \psi_{a}-\xi \frac{\left(\partial_{\mu} A^{\mu}\right)^{2}}{\sqrt{-\square}} \\
& -\sum_{l=1}^{8} \frac{G_{l}}{2}\left(\bar{\psi}_{a} \Gamma^{l} \psi_{a}\right)^{2},
\end{aligned}
$$

where $F_{\mu \nu}=\partial_{\mu} A_{\nu}-\partial_{\nu} A_{\mu}$ is the field intensity tensor of the gauge field $A_{\mu}, \square$ is the d'Alembertian operator, $\psi_{a}$ is the Dirac field, and $a=1, \ldots, N$ is the flavor index. For electrons in the honeycomb lattice, we may use the representation for matter field as $\psi_{a}^{\dagger}=\left(\psi_{A \uparrow}^{*}, \psi_{A \downarrow}^{*}, \psi_{B \uparrow}^{*}, \psi_{B \downarrow}^{*}\right)_{a}$, where $(A, B)$ and $(\uparrow, \downarrow)$ are the sublattices and spins, respectively. Therefore, one finds $a=K, K^{\prime}$ and $N=2$, which describes the valley degeneracy. Here, we perform all of the calculations for an arbitrary value of $N$ [21,22]. Furthermore, $m$ is the Dirac mass, $e$ is the electric charge, $\xi$ is the gauge-fixing parameter, $G_{l}=\left\{G_{1}, \ldots, G_{8}\right\}$ are the coupling constants of the four-fermion interactions where $l=1, \ldots, 8$ is an index describing each self-interaction, $\Gamma_{l}=\left\{\mathbb{1}, \gamma^{\mu}, \gamma^{3}, \gamma^{5}, \gamma^{\mu} \gamma^{3}, \gamma^{\mu} \gamma^{5}, \gamma^{3} \gamma^{5}, \gamma^{\mu} \gamma^{3} \gamma^{5}\right\} \quad$ are their corresponding matrices, $\gamma^{\mu}$ are the Dirac matrices in the $4 \times 4$ representation, whose algebra is given by $\left\{\gamma^{\mu}, \gamma^{\nu}\right\}=$ $-2 \delta^{\mu \nu}$, and $\gamma^{\mu} D_{\mu}=\gamma^{0} \partial_{0}+v_{F} \gamma^{i} \partial_{i}+e \gamma^{\mu} A_{\mu}$ is the Dirac operator after we perform the minimal coupling with $A_{\mu}$. Our matrix representation follows the definition given in Ref. [23]. Thus, our Dirac matrices are anti-Hermitian: $\left.\left(\gamma_{0}, \gamma_{1}, \gamma_{2}\right)=\left(i \sigma_{3}, i \sigma_{1}, i \sigma_{2}\right) \otimes, \sigma_{3}\right), \gamma_{3}=I \otimes \sigma_{1}$, and $\gamma_{5} \equiv$ $I \otimes \sigma_{2}$ so that $\left(\gamma_{\mu}\right)^{2}=-1$, and $\gamma_{5}$ is Hermitian. Furthermore, we shall use the natural system of units, where $\hbar=c=1$. Because $\left[G_{l}\right]=-1$, the model in Eq. (1) is not renormalizable in the perturbative expansion, but it is in the large- $N$ expansion. Hence, we shall consider the large- $N$ expansion from now on.

The first step is to introduce the $N$ parameter into the action through a scaling of the coupling constants, given by $e \rightarrow e / \sqrt{N}$ and $G_{l} \rightarrow G_{l} / N$ for a fixed $e$ and $G_{l}$, respectively. Thereafter, we use a Hubbard-Stratonovich transform in the four-fermion interactions, given by

$$
\begin{aligned}
\frac{G_{l}}{2 N}\left(\bar{\psi}_{a} \Gamma^{l} \psi_{a}\right)^{2} \rightarrow & \frac{G_{l}}{2 N}\left(\bar{\psi}_{a} \Gamma^{l} \psi_{a}\right)^{2} \\
& -\frac{N}{2 G_{1}}\left[\varphi_{1}-\frac{G_{1}}{N} \bar{\psi}_{a} \Gamma_{1} \psi_{a}\right]^{2}-\cdots \\
& -\frac{N}{2 G_{8}}\left[\varphi_{8}-\frac{G_{8}}{N} \bar{\psi}_{a} \Gamma_{8} \psi_{a}\right]^{2},
\end{aligned}
$$

where

$$
\varphi_{l}=\left\{\varphi_{1}, \varphi_{\gamma^{\mu}}, \varphi_{\gamma^{3}}, \varphi_{\gamma^{5}}, \varphi_{\gamma^{\mu} \gamma^{3}}, \varphi_{\gamma^{\mu} \gamma^{5}}, \varphi_{\gamma^{3} \gamma^{5}}, \varphi_{\gamma^{\mu} \gamma^{3} \gamma^{5}}\right\}
$$

is a set of auxiliary fields for each kind of interaction. Note that, for the sake of simplicity, we applied the notation $\varphi_{l}=\left\{\varphi_{1}, \ldots, \varphi_{8}\right\}$ in Eq. (2). Using Eq. (2) in Eq. (1), one finds the motion equation for the auxiliary fields, namely $\varphi_{l}=G_{l} \bar{\psi}_{a} \Gamma_{l} \psi_{a} / N$ at the classical level for each $l=$ $1, \ldots, 8$ (there is no sum over $l$ in the rhs of this equation). Furthermore, we also obtain the action,

$$
\begin{aligned}
\mathcal{L}= & \frac{1}{2} \frac{F^{\mu \nu} F_{\mu \nu}}{\sqrt{-\square}}+\bar{\psi}\left(i \gamma^{0} \partial_{0}+i v_{F} \gamma^{i} \partial_{i}-m\right) \psi \\
& +\frac{e}{\sqrt{N}} \bar{\psi} \gamma^{\mu} \psi A_{\mu}-\xi \frac{\left(\partial_{\mu} A^{\mu}\right)^{2}}{\sqrt{-\square}} \\
& +\sum_{l=1}^{8}\left[\frac{N}{2 G_{l}} \varphi_{l}^{2}-\varphi_{l} \bar{\psi} \Gamma_{l} \psi\right]
\end{aligned}
$$

Next, we realize a simple shift in the auxiliary field, namely $\varphi_{l} \rightarrow \sigma_{0, l}+\varphi_{l} / \sqrt{N}$, such that $\sigma_{0, l}=\left\langle\varphi_{l}\right\rangle$ is the vacuum expectation value of $\varphi_{l}$. Using this transform in Eq. (3), we have 


$$
\begin{aligned}
\mathcal{L}= & \frac{1}{2} \frac{F^{\mu \nu} F_{\mu \nu}}{\sqrt{-\square}}+\bar{\psi}\left(i \gamma^{0} \partial_{0}+i v_{F} \gamma^{i} \partial_{i}-m-\sigma_{0, l} \Gamma_{l}\right) \psi \\
& +\frac{e}{\sqrt{N}} \bar{\psi} \gamma^{\mu} \psi A_{\mu}-\xi \frac{\left(\partial_{\mu} A^{\mu}\right)^{2}}{\sqrt{-\square}}+\sum_{l=1}^{8}\left[\frac{N}{2 G_{l}} \sigma_{0, l}^{2}\right] \\
& +\sum_{l=1}^{8}\left[\frac{1}{2 G_{l}} \varphi_{l}^{2}-\frac{\sqrt{N}}{G_{l}} \sigma_{0, l} \varphi_{l}-\frac{1}{\sqrt{N}} \varphi_{l} \bar{\psi} \Gamma_{l} \psi\right] .
\end{aligned}
$$

One advantage of Eq. (4) is that for $m=0$, it clearly separates the analysis into phases, i.e., one with no spontaneous symmetry breaking where $\sigma_{0, l}=0$ and other phases with some broken symmetry $\sigma_{0, l} \neq 0$. In particular, a phase with chiral symmetry breaking, i.e., $\sigma_{0,1} \neq 0$, has been discussed in Ref. [16]. Next, let us define the Feynman rules. The gauge-field propagator in Eq. (3) reads

$$
\Delta_{\mu \nu}^{0}(p)=\frac{1}{2 \sqrt{p}}\left[\delta_{\mu \nu}-\left(1-\frac{1}{\xi}\right) \frac{p_{\mu} p_{\nu}}{p^{2}}\right]
$$

while the fermion propagator is given by

$$
S_{F}(p)=-\frac{1}{\gamma^{0} p_{0}+v_{F} \gamma^{i} p_{i}-m},
$$

and, in the tree approximation, the propagator for the auxiliary-field $\varphi_{l}$ is

$$
\left(\Delta_{\varphi_{l}}^{0}\right)=\left(\frac{1}{G_{l}}\right)^{-1}
$$

The electromagnetic and trilinear vertices interactions are given by $e / \sqrt{N}$ and $1 / \sqrt{N}$, respectively. Next, we shall calculate the quantum corrections, within the large- $N$ approximation, for the field propagators.

\section{FULL PROPAGATORS}

\section{A. Gauge-field propagator}

The full gauge-field propagator, in the dominant order of $1 / N$, is written as [16]

$$
\Delta_{\mu \nu}(p)=\Delta_{\mu \nu}^{0}(p)+\Delta_{\mu \alpha}^{0}(p) \Pi^{\alpha \beta}(p) \Delta_{\beta \nu}^{0}(p)+\cdots,
$$

where $\Pi^{\mu \nu}(p)$ is the vacuum polarization tensor, namely

$$
\Pi^{\mu \nu}(p)=-\frac{e^{2}}{N} \operatorname{Tr} \int \frac{d^{3} k}{(2 \pi)^{3}} \gamma^{\mu} S_{F}(p+k) \gamma^{\nu} S_{F}(k) .
$$

In the static limit, we only need the component $\Pi^{00}(p)$ given by

$$
\Pi^{00}\left(\mathbf{p}^{2}\right)=-\frac{e^{2}}{8} \frac{\mathbf{p}^{2}}{\sqrt{p_{0}^{2}+v_{F}^{2} \mathbf{p}^{2}}}
$$

in the small-mass limit $m^{2} \ll p^{2}$. Using Eq. (10) in Eq. (8), we find

$$
\Delta_{00}\left(\mathbf{p}^{2}\right)=\left(2 \sqrt{\mathbf{p}^{2}}+\frac{e^{2}}{8} \frac{\mathbf{p}^{2}}{\sqrt{p_{0}^{2}+v_{F}^{2} \mathbf{p}^{2}}}\right)^{-1} .
$$

This agrees with the result in Ref. [16].

\section{B. Auxiliary-field propagators}

The quantum corrections for the auxiliary fields $\varphi_{l}$ may be obtained through the effective action $S_{\text {eff }}$. This is accomplished from Eq. (4) by integrating out the matter field. After expanding $S_{\text {eff }}$ for large- $N$, we find

$$
S_{\mathrm{eff}}\left[\varphi_{l}\right]=\sqrt{N} S_{1}\left[\varphi_{l}\right]+S_{2}\left[\varphi_{l}\right]+\cdots,
$$

where

$$
\begin{aligned}
S_{1}= & \operatorname{Tr}\left[\left(i \gamma^{0} \partial_{0}+i v_{F} \gamma^{i} \partial_{i}-m-\sigma_{o, l} \Gamma^{l}\right)^{-1}\right. \\
& \left.\times\left(\sum_{l} \varphi_{l} \Gamma^{l}\right)\right]+\sum_{l} \frac{1}{G_{l}} \sigma_{0, l} \varphi_{l},
\end{aligned}
$$

and

$$
\begin{aligned}
S_{2}= & \frac{1}{2} \operatorname{Tr}\left[\left\{\left(i \gamma^{0} \partial_{0}+i v_{F} \gamma^{i} \partial_{i}-m-\sigma_{o, l} \Gamma^{l}\right)^{-1}\right.\right. \\
& \left.\left.\times\left(\sum_{l} \varphi_{l} \Gamma^{l}\right)\right\}^{2}\right]+\int d^{3} x \sum_{l} \frac{1}{2 G_{l}} \varphi_{l}^{2} .
\end{aligned}
$$

Note that $S_{1}$ in Eq. (13) may be written as $S_{1}=$ $\sum_{l} \varphi_{l} S_{l}\left[\sigma_{0, l}, G_{l}\right]$. On the other hand, we have that $S_{1}=0$, which implies a convergent effective action in Eq. (12). This yields a set of gap equations $S_{l}\left[\sigma_{0, l}, G_{l}\right]=0$ for each $l$, giving a nontrivial relation between the values of $\sigma_{0, l}$ and the coupling constants $G_{l}$. However, for $\sigma_{0, l}=0$ and $m=0$, these gap equations are automatically satisfied.

Next, it is convenient to write Eq. (14) as

$$
S_{2}=\frac{1}{2} \int d^{3} x d^{3} y \varphi_{l}(x) \Gamma^{l l^{\prime}}(x-y) \varphi_{l^{\prime}}(y),
$$

providing the auxiliary-field propagator $\Gamma^{l l^{\prime}}(x-y)^{-1}$. This, in the momentum space, is schematically written as

$$
\left[\Delta_{\left\{\varphi_{l}\right\}}^{l l^{\prime}}(p)\right]^{-1}=\Gamma_{\left\{\varphi_{l}\right\}}^{l l^{\prime}}(p)=\frac{1}{G_{\left\{\varphi_{l}\right\}}} \delta^{l l^{\prime}}-\Pi_{\left\{\varphi_{l}\right\}}^{l l^{\prime}}(p) .
$$

At this point, we must be careful with our notation in order to avoid any misunderstanding. Indeed, the kind of indexes $\left(l l^{\prime}\right)$ we have in Eq. (16) depends on the kind of auxiliary field $\left\{\varphi_{l}\right\}$ we want to consider. For instance, $\varphi_{1}=\varphi_{1} \rightarrow \sigma$ is a scalar field; hence, $\delta^{l^{\prime}}$ only means an unity. 


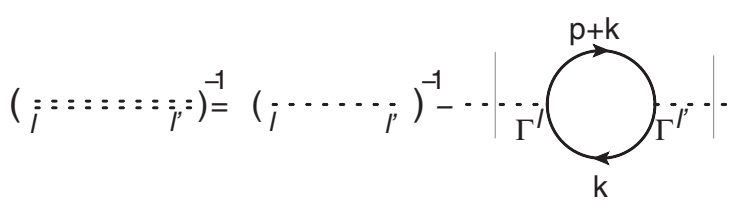

FIG. 1. The large- $N$ approximation for the full auxiliary-field propagators. The full propagators of the auxiliary fields are represented by the double-dashed line with the subscripts $\left(l, l^{\prime}\right)$, which are meant to describe their different tensorial structure. The continuum line is the propagator of the fermion field.

Nevertheless, we may consider the second auxiliary field, which is actually $\varphi_{2}=\varphi_{\gamma_{\mu}} \rightarrow \mathcal{A}_{\mu}$, a vector field. In this case, we must consider that $\delta^{l l^{\prime}} \rightarrow \delta^{\mu \nu}$, where we replace $\left(l l^{\prime}\right)$ by two Lorentz indexes, i.e., $\left(l l^{\prime}\right) \rightarrow(\mu \nu)$, such that we find a propagator $\Delta_{\left\{\varphi_{\mu}\right\}}^{\mu \nu}(p)$, as expected. The main rule is that for a generic auxiliary field $\left\{\varphi_{l}\right\}$, one must have a scalar quantity $S_{2} \propto \varphi_{l} \Gamma^{l l^{\prime}} \varphi_{l^{\prime}}$, which, therefore, fixes the tensorial structure of $\Gamma^{l l^{\prime}}$. We represent the full propagator of the auxiliary fields in Fig. 1.

The different self-energies for each auxiliary field read

$$
\Pi_{\left\{\varphi_{l}\right\}}^{l^{\prime}}(p)=-\operatorname{Tr} \int \frac{d^{3} k}{(2 \pi)^{3}} \Gamma^{l} S_{F}(p+k) \Gamma^{l^{\prime}} S_{F}(k) .
$$

We consider the $4 \times 4$ representation of the Dirac matrices, whose trace operations are detailed in the Appendix. Because of the Lorentz symmetry in the Dirac matrices, we perform a redefinition of the external momentum as $v_{F} p_{i} \rightarrow \bar{p}_{i}$, such that $\bar{p}_{\mu}=\left(p_{0}, \bar{p}_{i}\right)$. Furthermore, for the sake of consistency, we also change the spatial variable of the loop integral as $v_{F} k_{i} \rightarrow k_{i}$, which implies that $d^{3} k \rightarrow d^{3} k / v_{F}^{2}$. Therefore,

$\Pi_{\left\{\varphi_{l}\right\}}^{l l^{\prime}}(\bar{p})=-\frac{1}{v_{F}^{2}} \operatorname{Tr} \int \frac{d^{3} k}{(2 \pi)^{3}} \Gamma^{l} S_{F}(\bar{p}+k) \Gamma^{l^{\prime}} S_{F}(k)$.

It is clear that the only difference, between the different four-fermion interactions, is the vertex structure $\Gamma_{l}$ (and $\Gamma_{l^{\prime}}$ ) in Eq. (18).

\section{THE ELECTRON SELF-ENERGY}

We assume the symmetric phase, where $\sigma_{0, l}=0$. This phase is promptly obtained from Eq. (4) by using $\sigma_{0, l}=0$. Using the Feynman parametrization and the dimensional regularization (see the Appendix), we obtain the selfenergies for all of the auxiliary fields $\varphi_{l}$, which for higher momenta, is given by

$$
\begin{gathered}
\Gamma_{\varphi_{1}}(\bar{p})=\frac{1}{G_{\varphi_{1}}}+\frac{\sqrt{\bar{p}^{2}}}{4 v_{F}^{2}}, \\
\Gamma_{\varphi_{\mu}}^{\mu \nu}(\bar{p})=\left(\frac{1}{G_{\varphi_{\mu}}}+\frac{\sqrt{\bar{p}^{2}}}{8 v_{F}^{2}}\right) \overline{\mathbb{P}}^{\mu \nu}+\frac{1}{G_{\varphi_{\mu}}} \frac{\bar{p}^{\mu} \bar{p}^{\nu}}{\bar{p}^{2}},
\end{gathered}
$$

$$
\begin{gathered}
\Gamma_{\varphi_{3(5)}}(\bar{p})=\frac{1}{G_{\varphi_{3(5)}}}-\frac{\sqrt{\bar{p}^{2}}}{4 v_{F}^{2}}, \\
\Gamma_{\varphi_{\mu 3(5)} \nu}(\bar{p})=\left(\frac{1}{G_{\varphi_{\mu 3(5)}}}+\frac{\sqrt{\bar{p}^{2}}}{8 v_{F}^{2}}\right) \overline{\mathbb{P}}^{\mu \nu}+\frac{1}{G_{\varphi_{\mu 3(5)}}} \frac{\bar{p}^{\mu} \bar{p}^{\nu}}{\bar{p}^{2}}, \\
\Gamma_{\varphi_{35}}(\bar{p})=\frac{1}{G_{\varphi_{35}}}+\frac{\sqrt{\bar{p}^{2}}}{4 v_{F}^{2}},
\end{gathered}
$$

and

$$
\Gamma_{\varphi_{\mu 35}}^{\mu \nu}(\bar{p})=\left(\frac{1}{G_{\varphi_{\mu 35}}}-\frac{\sqrt{\bar{p}^{2}}}{8 v_{F}^{2}}\right) \overline{\mathbb{P}}^{\mu \nu}+\frac{1}{G_{\varphi_{\mu 35}}} \frac{\bar{p}^{\mu} \bar{p}^{\nu}}{\bar{p}^{2}} .
$$

The subscription 3(5) means that the result holds for both $\varphi_{3}$ and $\varphi_{5}$ fields, for example. Furthermore, the standard projection tensor $\overline{\mathbb{P}}^{\mu \nu}$ reads

$$
\overline{\mathbb{P}}^{\mu \nu}=\delta^{\mu \nu}-\frac{\bar{p}^{\mu} \bar{p}^{\nu}}{\bar{p}^{2}} .
$$

It should be noticed the bad ultraviolet behavior of the longitudinal part of the two point proper function involving a vectorial field, namely the longitudinal parts in Eqs. (20), (22), and (24). Of course, these bad behaviors are innocuous if the corresponding currents are conserved. In any case, this fact is only relevant for calculating the correction in order $1 / N^{2}$. If we consider only the transversal part of these propagators, the generalized model (3) is power counting renormalizable with divergences being eliminated by reparametrizations of the fields and of the mass of the fermion field. In what follows, we will discuss in detail the divergences in the fermion self-energy.

\section{A. The fermion self-energy}

Having the gauge and auxiliary-field propagators, we may calculate the fermion self-energy. This also may be decomposed into two terms, one due to the gauge field and the other due to the auxiliary fields.

\section{Self-energy due to the gauge field}

The fermion self-energy due to the gauge field is shown in Fig 2(a), and its analytical expression is given by

$$
\Sigma_{A_{\mu}}(p)=\frac{e^{2}}{N} \int \frac{d^{3} k}{(2 \pi)^{3}} \gamma^{\mu} S_{F}(p-k) \gamma^{\nu} \Delta_{\mu \nu}(k) .
$$

The first step is to use Eq. (6) and Eq. (11) in Eq. (26). On the other hand, the self-energy in the small-momentum limit, which is the relevant term in order to extract the form of the divergences, is written as $[16,24]$ 


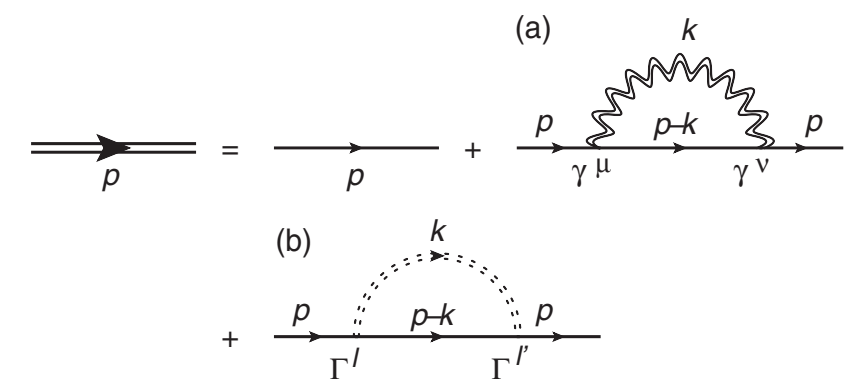

FIG. 2. The full fermion propagator up to the dominant order $1 / N$. The full fermion propagator is represented by the double continuous lines. (a) The self-energy due to the interaction between the gauge and fermion fields. (b) The general structure of the self-energies due to each auxiliary-field propagator and the fermion field.

$$
\begin{aligned}
\Sigma_{A_{\mu}}(p)= & \left.\Sigma_{A_{\mu}}(p)\right|_{p=0}+\left.\gamma^{0} p_{0} \frac{\partial \Sigma_{A_{\mu}}\left(p_{0}\right)}{\partial p_{0}}\right|_{p_{0}=0} \\
& +\left.v_{F} \gamma^{i} p_{i} \frac{\partial \Sigma_{A_{\mu}}\left(p_{i}\right)}{\partial p_{i}}\right|_{p_{i}=0}+\cdots
\end{aligned}
$$

After some calculations (see Appendix A of Ref. [16]), it is possible to show that the fermion self-energy, in the smallmass limit, is

$$
\begin{aligned}
\Sigma_{A_{\mu}}(p)= & -\frac{2 \lambda}{\pi^{2} N}\left[\gamma^{0} p_{0} f_{1}(\lambda)-v_{F} \gamma^{i} p_{i} f_{2}(\lambda)\right. \\
& \left.+m f_{0}(\lambda)\right] \ln \left(\frac{\Lambda}{\Lambda_{0}}\right)+\mathrm{FT},
\end{aligned}
$$

where FT stands for finite terms, $\lambda=e^{2} /\left(16 v_{F}\right)=\pi \alpha / 4$, where $\alpha$ is the fine-structure constant,

$$
\begin{gathered}
f_{0}(\lambda)=\frac{2 \cos ^{-1}(\lambda)}{\sqrt{1-\lambda}}, \\
f_{1}(\lambda)=-\frac{2}{\lambda^{2}}\left[\pi-2 \lambda+\frac{\left(\lambda^{2}-2\right)}{\sqrt{1-\lambda^{2}}} \cos ^{-1}(\lambda)\right],
\end{gathered}
$$

and

$$
f_{2}(\lambda)=\frac{1}{\lambda^{2}}\left[\pi-2 \lambda-2 \sqrt{1-\lambda^{2}} \cos ^{-1}(\lambda)\right] .
$$

\section{Self-energy due to the auxiliary fields}

The fermion self-energy due to the auxiliary fields is shown in Fig. 2(b), and its analytical expression is given by

$$
\Sigma_{\left\{\varphi_{l}\right\}}(p)=\int \frac{d^{3} k}{(2 \pi)^{3}} \Gamma^{l} S_{F}(p-k) \Gamma^{l^{\prime}} \Delta_{l l^{\prime}}^{\left\{\varphi_{l}\right\}}(k) .
$$

Here, we use Eq. (6) and Eq. (16) in Eq. (32). Thereafter, we make the same reassignement of the momentum variables as before; i.e., we redefine the external momentum as $p_{\mu} \rightarrow \bar{p}_{\mu}$, where $\bar{p}_{\mu}=\left(p_{0}, \bar{p}_{i}\right)$, and change the loop-integral variable as $v_{F} k_{i} \rightarrow k_{i}$. Therefore, the selfenergy is written as

$$
\Sigma_{\left\{\varphi_{l}\right\}}(\bar{p})=\frac{1}{v_{F}^{2}} \int \frac{d^{3} k}{(2 \pi)^{3}} \Gamma^{l} \frac{\gamma^{\alpha}(\bar{p}-k)_{\alpha}+m}{(\bar{p}-k)^{2}+m^{2}} \Gamma^{l^{\prime}} \Delta_{l l^{\prime}}^{\left\{\varphi_{l}\right\}}(k) .
$$

Similarly to the previous case, we expand the self-energy as

$$
\Sigma_{\left\{\varphi_{l}\right\}}(\bar{p})=\left.\Sigma_{\left\{\varphi_{l}\right\}}(\bar{p})\right|_{\bar{p}=0}+\left.\bar{p}_{\mu} \frac{\partial \Sigma_{\left\{\varphi_{l}\right\}}(\bar{p})}{\partial \bar{p}_{\mu}}\right|_{\bar{p}=0}+\cdots
$$

Note that the lowest-order term in Eq. (34) is

$$
\left.\Sigma_{\left\{\varphi_{l}\right\}}(\bar{p})\right|_{\bar{p}=0}=\frac{m}{v_{F}^{2}} \int \frac{d^{3} k}{(2 \pi)^{3}} \frac{\Gamma^{l} \Gamma^{l^{\prime}}}{k^{2}+m^{2}} \Delta_{l l^{\prime}}^{\left\{\varphi_{l}\right\}}(k),
$$

and the first-order term in $\bar{p}_{\mu}$ reads

$$
\begin{aligned}
\left.\bar{p}_{\mu} \frac{\partial \Sigma_{\left\{\varphi_{l}\right\}}(\bar{p})}{\partial \bar{p}_{\mu}}\right|_{\bar{p}=0}= & \frac{1}{v_{F}^{2}} \int \frac{d^{3} k}{(2 \pi)^{3}} \Gamma^{l}\left\{\frac{\gamma^{\mu} \bar{p}_{\mu}}{k^{2}+m^{2}}\right. \\
& \left.-2 \frac{\gamma^{\alpha} k_{\alpha} k^{\mu} \bar{p}_{\mu}}{\left[k^{2}+m^{2}\right]^{2}}\right\} \Gamma^{l^{\prime}} \Delta_{l l^{\prime}}^{\{\varphi\}}(k) .
\end{aligned}
$$

For analyzing the divergent parts of these expressions, we may neglect the $1 / G_{\varphi_{l}}$ terms in the propagators of the auxiliary fields as they only give finite contributions. We assume that $1 / G_{\varphi_{l}} \ll \sqrt{\bar{p}^{2}}$ as an approximation in the auxiliary-field propagators for calculating the fermion selfenergy. Let us take the Thirring interaction as a concrete example; hence, $\left\{\varphi_{l}\right\} \rightarrow \varphi_{2}=\varphi_{\gamma_{\mu}}$. In this case, the zeroorder term reads

$$
\begin{aligned}
\left.\Sigma_{\varphi_{\mu}}(\bar{p})\right|_{\bar{p}=0}= & \frac{8}{N} m \int \frac{d^{3} k}{(2 \pi)^{3}}\left\{\frac{\gamma^{\mu} \gamma_{\mu}}{\left(k^{2}+m^{2}\right)}\right. \\
& \left.-\frac{\gamma^{\mu} \gamma^{\nu} k_{\mu} k_{\nu}}{\left(k^{2}+m^{2}\right) k^{2}}\right\} \frac{1}{\sqrt{k^{2}}} .
\end{aligned}
$$

Next, we use $\gamma^{\mu} \gamma_{\mu}=-3$ and, given the Lorentz invariance on the integral, we change $k_{\mu} k_{\nu} \rightarrow g_{\mu \nu} k^{2} / 3$. Using these conditions, Eq. (37) yields

$$
\left.\Sigma_{\varphi_{\mu}}(\bar{p})\right|_{\bar{p}=0}=-\frac{16}{N} m \int \frac{d^{3} k}{(2 \pi)^{3}} \frac{1}{k^{2}+m^{2}} \frac{1}{\sqrt{k^{2}}} .
$$

After applying the Feynman parametrization and cut-off regularization, within the small-mass limit, we find the zero-order term, namely 


$$
\left.\Sigma_{\varphi_{\mu}}(\bar{p})\right|_{\bar{p}=0}=-\frac{8}{\pi^{2} N} m \ln \left(\frac{\Lambda}{\Lambda_{0}}\right)
$$

Next, let us calculate the first-order term. From the expansion given by Eq. (36), we find

$$
\begin{aligned}
\left.\bar{p}_{\beta} \frac{\Sigma_{\varphi_{\mu}}(\bar{p})}{\partial \bar{p}_{\beta}}\right|_{\bar{p}=0}= & \frac{8}{N} \int \frac{d^{3} k}{(2 \pi)^{3}} \gamma^{\mu}\left\{\frac{\gamma^{\beta} \bar{p}_{\beta}}{k^{2}+m^{2}}\right. \\
& \left.-2 \frac{\gamma^{\alpha} k_{\alpha} k^{\beta} \bar{p}_{\beta}}{\left(k^{2}+m^{2}\right)^{2}}\right\} \gamma^{\nu} \frac{\mathbb{K}_{\mu \nu}}{\sqrt{k^{2}}} .
\end{aligned}
$$

We shall follow the same steps as before. Here, however, for the second integral in rhs of Eq. (40), we use $k_{\alpha} k^{\beta} k_{\mu} k_{\nu} \rightarrow\left(\delta_{\alpha}^{\beta} \delta_{\mu \nu}+\delta_{\alpha \mu} \delta_{\nu}^{\beta}+\delta_{\alpha \nu} \delta_{\mu}^{\beta}\right) k^{4} / 15$, because of the Lorentz invariance in the loop integral. Therefore, we obtain

$$
\left.\bar{p}_{\beta} \frac{\partial \Sigma_{\varphi_{\mu}}(\bar{p})}{\partial \bar{p}_{\beta}}\right|_{\bar{p}=0}=-\frac{8}{3 \pi^{2} N} \gamma^{\beta} \bar{p}_{\beta} \ln \left(\frac{\Lambda}{\Lambda_{0}}\right)+\mathrm{FT},
$$

with being FT the finite terms. From Eq. (39) and Eq. (41), we find the whole contribution of the Thirring interaction to the fermion self-energy, given by

$$
\Sigma_{\varphi_{\mu}}(\bar{p})=-\frac{8}{3 \pi^{2} N}\left\{\gamma^{\mu} \bar{p}_{\mu}+3 m\right\} \ln \left(\frac{\Lambda}{\Lambda_{0}}\right)+\mathrm{FT} .
$$

After doing the same procedure for the other interactions, we find

$$
\begin{gathered}
\Sigma_{\varphi_{1}}(\bar{p})=\frac{2}{3 \pi^{2} N}\left\{\gamma^{\mu} \bar{p}_{\mu}+3 m\right\} \ln \left(\frac{\Lambda}{\Lambda_{0}}\right)+\mathrm{FT}, \\
\Sigma_{\varphi_{3(5)}}(\bar{p})=\frac{2}{3 \pi^{2} N}\left\{\gamma^{\mu} \bar{p}_{\mu}-3 m\right\} \ln \left(\frac{\Lambda}{\Lambda_{0}}\right)+\mathrm{FT}, \\
\Sigma_{\varphi_{\mu 3(5)}}(\bar{p})=-\frac{8}{3 \pi^{2} N}\left\{\gamma^{\mu} \bar{p}_{\mu}-3 m\right\} \ln \left(\frac{\Lambda}{\Lambda_{0}}\right)+\mathrm{FT}, \\
\Sigma_{\varphi_{35}}(\bar{p})=-\frac{2}{3 \pi^{2} N}\left\{\gamma^{\mu} \bar{p}_{\mu}+3 m\right\} \ln \left(\frac{\Lambda}{\Lambda_{0}}\right)+\mathrm{FT},
\end{gathered}
$$

and

$$
\Sigma_{\varphi_{\mu 35}}(\bar{p})=-\frac{8}{3 \pi^{2} N}\left\{\gamma^{\mu} \bar{p}_{\mu}+3 m\right\} \ln \left(\frac{\Lambda}{\Lambda_{0}}\right)+\mathrm{FT} .
$$

\section{RENORMALIZATION GROUP}

In general grounds, the renormalization group equation has so many anomalous dimensions as fields in the lagrangian. However, because the vacuum polarization tensors $\Pi^{\mu \nu}(p)$ and $\Pi_{\varphi_{l}}^{l l^{\prime}}(p)$ are finite in the dimensional regularization scheme, we conclude that $\gamma_{A_{\mu}}, \beta_{e}$, and $\gamma_{\varphi_{l}}$ vanish. Furthermore, the beta functions for the coupling constants $\beta_{G}$ do not appear in our renormalization group equation due to the approximation $1 / G_{l} \ll \sqrt{p^{2}}$ we have considered before. Having these assumptions in mind, our renormalization group equation is written as

$$
\left[\Lambda \frac{\partial}{\partial \Lambda}+\beta_{v_{F}} \frac{\partial}{\partial v_{F}}+\beta_{m} \frac{\partial}{\partial m}-N_{F} \gamma_{F}\right] \Gamma^{\left(N_{F}, \cdots\right)}=0,
$$

where $\Gamma^{N_{F}, \ldots}=\Gamma^{\left(N_{F}, N_{A}, N_{\varphi_{l}}\right)}\left(p_{1}, \ldots, p_{N}\right)$ are the renormalized vertex functions, and $\left(N_{F}, N_{A}, N_{\varphi_{l}}\right)$ are the number of external lines of fermion, gauge, and auxiliary fields, respectively. The beta functions of $v_{F}$ and $m$ parameters are $\beta_{v_{F}}=\Lambda \frac{\partial v_{F}}{\partial \Lambda}$ and $\beta_{m}=\Lambda \frac{\partial m}{\partial \Lambda}$, respectively. The anomalous dimension of the fermion field is $\gamma_{F}=\Lambda \frac{\partial}{\partial \Lambda}\left(\ln Z_{\psi}\right)$, where $Z_{\psi}$ is the wave function renormalization.

The two-point function for the fermion field is

$$
\Gamma^{(2)}=\left(\gamma^{0} p_{0}+v_{F} \gamma^{i} p_{i}-m\right)+\Sigma_{A_{\mu}}(p)+\Sigma_{l}^{\left\{\varphi_{l}\right\}}(p),
$$

where the contribution of the gauge field $\Sigma_{A_{\mu}}(p)$ is

$$
\Sigma_{A_{\mu}}(p)=\left[a_{1} \gamma^{0} p_{0}+a_{2} v_{F} \gamma^{i} p_{i}+a_{3} m\right] \ln \left(\frac{\Lambda}{\Lambda_{0}}\right),
$$

and the coefficients $a_{1,2,3}$ are easily obtained from Eq. (28). After recovering $\bar{p} \rightarrow p=p_{0}+v_{F} p_{i}$, we find the contribution of the auxiliary fields; i.e.,

$$
\Sigma_{l}^{\left\{\varphi_{l}\right\}}(p)=\left[b_{1}^{\left\{\varphi_{l}\right\}} \gamma^{0} p_{0}+b_{1}^{\left\{\varphi_{l}\right\}} v_{F} \gamma^{i} p_{i}+b_{2}^{\left\{\varphi_{l}\right\}} m\right] \ln \left(\frac{\Lambda}{\Lambda_{0}}\right)
$$

where the coefficients $b_{1,2}$ are obtained from Eq. (42), (43), and (44). In the large- $N$ expansion, we may write the beta functions as $\beta_{a}=\beta_{a}^{(1)}+\frac{1}{N} \beta_{a}^{(2)}+\cdots$, with $a=v_{F}, m$, and the anomalous dimension as $\gamma_{F}=\gamma_{F}^{(1)}+\frac{1}{N} \gamma_{F}^{(2)}+\cdots$. Thereafter, we replace Eq. (46) in Eq. (45) and, after some algebra, we obtain

$$
\begin{gathered}
\gamma_{F}=\frac{1}{2}\left(a_{1}+\sum_{l} b_{1}^{\left\{\varphi_{l}\right\}}\right), \\
\beta_{v_{F}}=v_{F}\left(a_{1}-a_{2}\right),
\end{gathered}
$$

and

$$
\beta_{m}=m\left[a_{1}+a_{3}+\sum_{l}\left(b_{1}^{\left\{\varphi_{l}\right\}}+b_{2}^{\left\{\rho_{l}\right\}}\right)\right] .
$$

Using the coefficients $a_{1,2,3}$ and $b_{1,2}$, we obtain 


$$
\begin{gathered}
\gamma_{F}=-\frac{2}{\pi^{2} N}\left[2+\frac{2-\lambda^{2}}{\lambda \sqrt{1-\lambda^{2}}} \cos ^{-1}(\lambda)-\frac{\pi}{\lambda}\right]-\frac{28}{3 \pi^{2} N}, \\
\beta_{v_{F}}=-\frac{4}{\pi^{2} N} v_{F}\left[1+\frac{\cos ^{-1}(\lambda)}{\lambda \sqrt{1-\lambda^{2}}}-\frac{\pi}{2 \lambda}\right],
\end{gathered}
$$

and

$$
\beta_{m}=-\frac{2 m}{\pi^{2} N}\left[4+\frac{4 \cos ^{-1}(\lambda)}{\lambda \sqrt{1-\lambda^{2}}}-\frac{2 \pi}{\lambda}\right]+\sum_{\left\{b_{4 F}\right\}} m b_{4 F},
$$

where $b_{4 F} \equiv b_{1}^{\left\{\varphi_{l}\right\}}+b_{2}^{\left\{\varphi_{l}\right\}}$ is the contribution, due to the four-fermion interactions, for the beta function of the mass. These are, in principle, different for each $\bar{\psi} \Gamma_{l} \psi$ term. Notice, however, that they do not depend on the couplings $1 / G_{l}$. In fact, by considering the high momenta expansions for the auxiliary field propagators, we may verify that terms containing these parameters are actually finite. In Table I, we summarize all of the possible values of $b_{4 F}$ generated by each individual interaction.

\section{A. Mass renormalization}

We obtain the renormalized mass through the beta function as

$$
\Lambda \frac{\partial m}{\partial \Lambda}=\beta_{m}
$$

with Eq. (54), where the renormalized mass depends on the energy scale $\Lambda$. After solving Eq. (55) for $m(\Lambda)$, it follows that

$$
m(\Lambda)=m\left(\Lambda_{0}\right)\left(\frac{\Lambda}{\Lambda_{0}}\right)^{g(\lambda)}
$$

where

TABLE I. The $b_{4 F}$ term of each four-fermion interaction. This table gives the contribution of each four-fermion interaction to the beta function of the mass, given in Eq. (51) by $b_{1}^{\left\{\varphi_{l}\right\}}+b_{2}^{\left\{\varphi_{l}\right\}}$. These contributions are calculated in the small-mass limit, where $m^{2} \ll p^{2}$ and for $1 / G_{l} \ll p$.

\begin{tabular}{lc}
\hline \hline The four-fermion interactions & The contribution $b_{4 F}$ \\
\hline$(\bar{\psi} \psi)^{2}$ & $8 /\left(3 \pi^{2} N\right)$ \\
$\left(\bar{\psi} \gamma \gamma^{\mu} \psi\right)^{2}$ & $-32 /\left(3 \pi^{2} N\right)$ \\
$\left(\bar{\psi} \gamma^{3} \psi\right)^{2}$ & $-4 /\left(3 \pi^{2} N\right)$ \\
$\left(\bar{\psi} \gamma^{5} \psi\right)^{2}$ & $-4 /\left(3 \pi^{2} N\right)$ \\
$\left(\bar{\psi} \gamma^{3} \gamma^{5} \psi\right)^{2}$ & $-8 /\left(3 \pi^{2} N\right)$ \\
$\left(\bar{\psi} \gamma \gamma^{\mu} \gamma^{3} \psi\right)^{2}$ & $16 /\left(3 \pi^{2} N\right)$ \\
$\left(\bar{\psi} \gamma^{\mu} \gamma^{5} \psi\right)^{2}$ & $16 /\left(3 \pi^{2} N\right)$ \\
$\left(\bar{\psi} \gamma \gamma^{\mu} \gamma^{3} \gamma^{5} \psi\right)^{2}$ & $-32 /\left(3 \pi^{2} N\right)$ \\
\hline \hline
\end{tabular}

$$
g(\lambda)=-\frac{2}{\pi^{2} N}\left[4+\frac{4 \cos ^{-1}(\lambda)}{\lambda \sqrt{1-\lambda^{2}}}\right]+\sum_{\left\{b_{4 F}\right\}} b_{4 F} .
$$

From Eq. (56) and Eq. (57), we conclude that the contribution of the $b_{4 F}$ terms, generated by the fourfermion interactions, modifies the behavior of the renormalized mass, because they may change the sign of the function $g(\lambda)$, as shown in Fig. 3. In Fig. 4, we plot the function $m(\Lambda)$. In general, there are three possible cases, namely (A) $g(\lambda)<0$ for any $\lambda$, (B) $g(\lambda)>0$ for any $\lambda$, and (C), where either $g(\lambda)>0$ for $\lambda>\lambda_{c}$ or $g(\lambda)<0$ for $\lambda<\lambda_{c}$. The critical point $\lambda_{c}$ is obtained from $g\left(\lambda_{c}\right)=0$. Obviously, only the case (C) allow us to control the renormalized mass by tuning the value of $\lambda$.

Next, let us consider the case (A). This is the regime where the renormalized mass is fully controlled by the electromagnetic interactions. Therefore, the sum over the $b_{4 F}$ term vanishes. In Fig. 5, we show a plot for such possibility. From Table I, we conclude that there are six different combinations that fulfil this criteria. These are (A1) $\quad(\bar{\psi} \psi)^{2}+\left(\bar{\psi} \gamma^{3} \gamma^{5} \psi\right)^{2}, \quad(\mathrm{~A} 2) \quad(\bar{\psi} \psi)^{2}+\left(\bar{\psi} \gamma \gamma^{3} \psi\right)^{2}+$ $\left(\bar{\psi} \gamma^{5} \psi\right)^{2}$, (A3) $\left(\bar{\psi} \gamma^{\mu} \psi\right)^{2}+\left(\bar{\psi} \gamma^{\mu} \gamma^{3} \psi\right)^{2}+\left(\bar{\psi} \gamma^{\mu} \gamma^{5} \psi\right)^{2}$ [where $\left(\bar{\psi} \gamma^{\mu} \psi\right)^{2}$ can be replaced by $\left.\left(\bar{\psi} \gamma^{\mu} \gamma^{3} \gamma^{5} \psi\right)^{2}\right]$, and (A4) $\left(\bar{\psi} \gamma^{\mu} \gamma^{3} \psi\right)^{2}+\left(\bar{\psi} \gamma^{3} \gamma^{5} \psi\right)^{2}+\left(\bar{\psi} \gamma^{3} \psi\right)^{2}+\left(\bar{\psi} \gamma \gamma^{5} \psi\right)^{2} \quad$ [where $\left(\bar{\psi} \gamma^{\mu} \gamma^{3} \psi\right)^{2}$ can be replaced by $\left.\left(\bar{\psi} \gamma^{\mu} \gamma^{5} \psi\right)^{2}\right]$. In case (A), we conclude that $m(\Lambda) \rightarrow 0$ as $\Lambda \rightarrow \infty$.

In case (B), we need combinations that always provide $g(\lambda)$ positive. In this regime, the influence of the fourfermion interactions is dominant over the contribution of

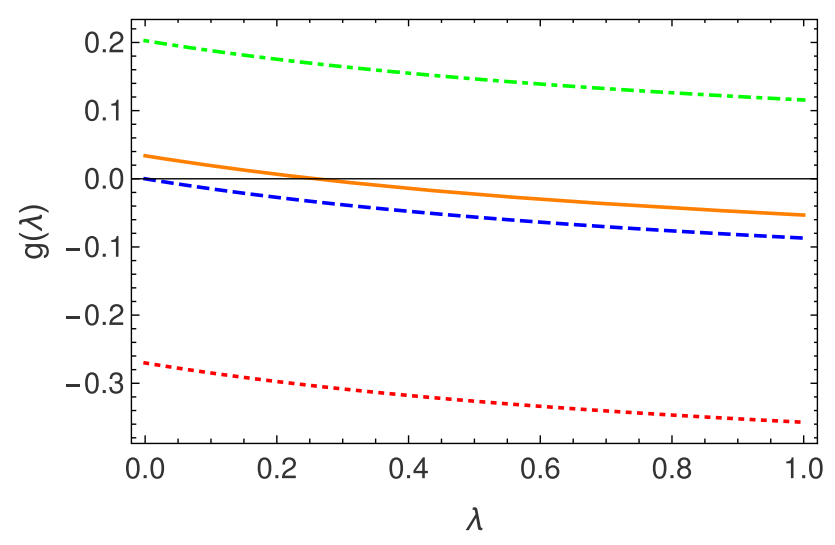

FIG. 3. The function $g(\lambda)$ in the interval $\lambda \in[0.1,1]$ with $N=4$. We plot Eq. (57) for four different combinations of the four-fermion interactions. The continuous line is obtained from the $(\bar{\psi} \psi)^{2}$ and $\left(\bar{\psi} \gamma^{3} \psi\right)^{2}$ interactions, which provides $\sum_{\left\{b_{4 F}\right\}} b_{4 F}=1 / 3 \pi^{2}$. In this case, we find $\lambda_{c}^{\min }=0.26$. The dashed line is the combination of $(\bar{\psi} \psi)^{2}$ and $\left(\bar{\psi} \gamma^{3} \gamma^{5} \psi\right)^{2}$ interactions, where $\sum_{\left\{b_{4 F}\right\}} b_{4 f}=0$. The dotted line is obtained from the $\left(\bar{\psi} \gamma^{\mu} \psi\right)^{2}$ interaction, where $b_{4 F}=-8 / 3 \pi^{2}$. The dasheddotted line is obtained from the $(\bar{\psi} \psi)^{2}$ and $\left(\bar{\psi} \gamma^{\mu} \gamma^{3} \psi\right)^{2}$ interactions, where $\sum_{\left\{b_{4 F}\right\}} b_{4 F}=2 / \pi^{2}$. Note that only for the continuous line, we have a critical point. 


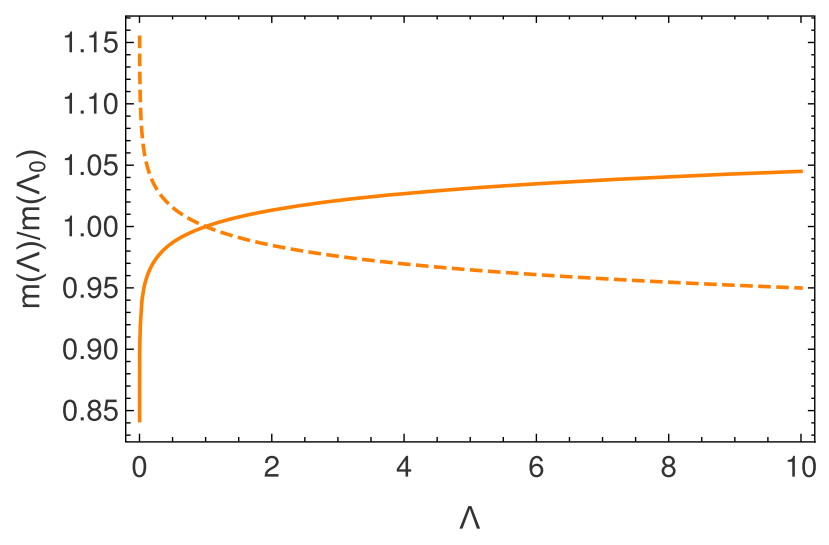

FIG. 4. The behavior of $m(\Lambda)$. We plot Eq. (56) considering the $(\bar{\psi} \psi)^{2}$ and $\left(\bar{\psi} \gamma^{3} \psi\right)^{2}$ interactions, which provides $\lambda_{c}^{\min }=0.26$. For the line, we use $\lambda=0.1$, while for the dashed line, we use $\lambda=0.5$. We consider $N=4$ for both curves.

the electromagnetic interactions. Therefore, the sum over the $b_{4 F}$ term must be larger than the first term in the rhs of Eq. (57) for any $\lambda$. In Fig. 5, we show a plot for such possibility. From Table I, we conclude that there are seven different combinations that fulfil this criteria. These are (B1) $\left(\bar{\psi} \gamma^{\mu} \gamma^{3} \psi\right)^{2}+\left(\bar{\psi} \gamma^{\mu} \gamma^{5} \psi\right)^{2}$, (B2) $(\bar{\psi} \psi)^{2}+\left(\bar{\psi} \gamma^{\mu} \gamma^{3} \psi\right)^{2}$ [where $\left(\bar{\psi} \gamma^{\mu} \gamma^{3} \psi\right)^{2}$ can be replaced by $\left(\bar{\psi} \gamma^{\mu} \gamma^{5} \psi\right)^{2}$ ], (B3) $\left(\bar{\psi} \gamma^{\mu} \gamma^{3} \psi\right)^{2}+\left(\bar{\psi} \gamma^{3} \psi\right)^{2}$, and (B4) $\left(\bar{\psi} \gamma^{\mu} \gamma^{5} \psi\right)^{2}+\left(\bar{\psi} \gamma^{3} \psi\right)^{2}$ [in the last two combinations, $\left(\bar{\psi} \gamma^{3} \psi\right)^{2}$ can replaced by $\left.\left(\bar{\psi} \gamma^{5} \psi\right)^{2}\right]$. In case (B), we conclude that $m(\Lambda) \rightarrow \infty$ as $\Lambda \rightarrow \infty$.

In case (C), the sign of $g(\lambda)$ changes after crossing the point $\lambda_{c}$. In this regime, the renormalized mass is described by the competition of electromagnetic and fourfermion interactions, where both of them are relevant. We find two possible values for the critical coupling constant,

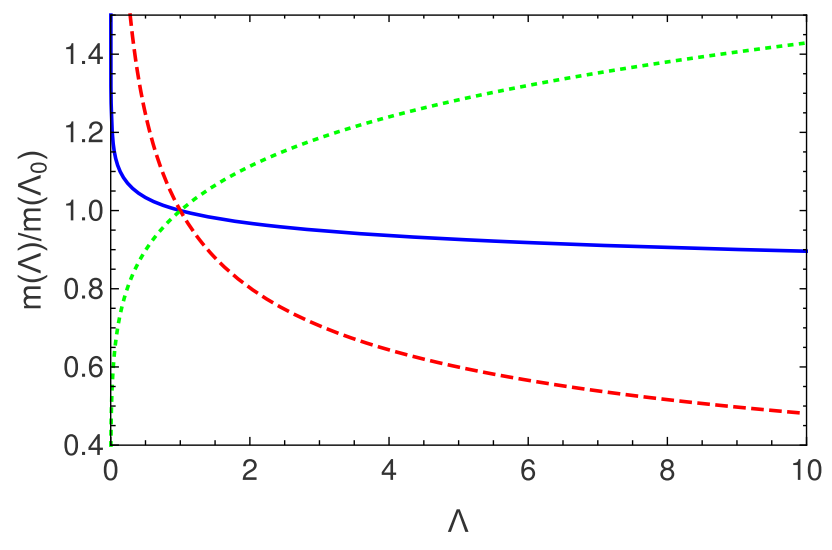

FIG. 5. The behavior of $m(\Lambda)$. We plot Eq. (56) with $N=4$ and $\lambda=0.4$. The continuous line is obtained from the $(\bar{\psi} \psi)^{2}$ and $\left(\bar{\psi} \gamma^{3} \gamma^{5} \psi\right)^{2}$ interactions (the same curve holds when we replace $\gamma_{3}$ by $\gamma_{5}$ ). The dashed line is obtained from the Thirring interaction $\left(\bar{\psi} \gamma^{\mu} \psi\right)^{2}$. The dotted line is obtained from $(\bar{\psi} \psi)^{2}$ and $\left(\bar{\psi} \gamma^{\mu} \gamma^{3} \psi\right)^{2}$ interactions. namely $\lambda_{c}^{\max }=0.66$ and $\lambda_{c}^{\min }=0.26$. From Table I, we find seven combinations that provide $\lambda_{c}^{\max }$, given by $(\mathrm{C} 1 \mathrm{~A})$ $(\bar{\psi} \psi)^{2}, \quad(\mathrm{C} 2 \mathrm{~A})\left(\bar{\psi} \gamma^{\mu} \gamma^{3} \psi\right)^{2}+\left(\bar{\psi} \gamma^{3} \gamma^{5} \psi\right)^{2}, \quad(\mathrm{C} 3 \mathrm{~A})(\bar{\psi} \psi)^{2}+$ $\left(\bar{\psi} \gamma^{\mu} \gamma^{3} \psi\right)^{2}+\left(\bar{\psi} \gamma^{3} \gamma^{5} \psi\right)^{2}+\left(\bar{\psi} \gamma^{3} \psi\right)^{2}+\left(\bar{\psi} \gamma^{5} \psi\right)^{2}$, and (C4A) $\left(\bar{\psi} \gamma^{\mu} \gamma^{3} \psi\right)^{2}+\left(\bar{\psi} \gamma^{3} \psi\right)^{2}+\left(\bar{\psi} \gamma^{5} \psi\right)^{2}$ [in each of the previous combinations, we can change $\left(\bar{\psi} \gamma^{\mu} \gamma^{3} \psi\right)^{2}$ by $\left.\left(\bar{\psi} \gamma^{\mu} \gamma^{5} \psi\right)^{2}\right]$. On the other hand, for finding $\lambda_{c}^{\min }$, there are six possibilities, namely (C1B) $(\bar{\psi} \psi)^{2}+\left(\bar{\psi} \gamma^{3} \psi\right)^{2}$ and (C2B) $(\bar{\psi} \psi)^{2}+\left(\bar{\psi} \gamma^{5} \psi\right)^{2}, \quad(\mathrm{C} 3 \mathrm{~B}) \quad\left(\bar{\psi} \gamma^{\mu} \gamma^{3} \psi\right)^{2}+\left(\bar{\psi} \gamma^{3} \gamma^{5} \psi\right)^{2}+$ $\left(\bar{\psi} \gamma^{3} \psi\right)^{2}, \quad(\mathrm{C} 4 \mathrm{~B}) \quad\left(\bar{\psi} \gamma^{\mu} \gamma^{3} \psi\right)^{2}+\left(\bar{\psi} \gamma^{3} \gamma^{5} \psi\right)^{2}+\left(\bar{\psi} \gamma^{5} \psi\right)^{2}$, (C5B) $\left(\bar{\psi} \gamma^{\mu} \gamma^{5} \psi\right)^{2}+\left(\bar{\psi} \gamma^{3} \gamma^{5} \psi\right)^{2}+\left(\bar{\psi} \gamma^{3} \psi\right)^{2}$, and (C6B) $\left(\bar{\psi} \gamma^{\mu} \gamma^{5} \psi\right)^{2}+\left(\bar{\psi} \gamma \gamma^{3} \gamma^{5} \psi\right)^{2}+\left(\bar{\psi} \gamma^{5} \psi\right)^{2}$. Case (C) clearly provides two possible asymptotic behaviors for $m(\Lambda)$; see Fig. 5.

In Ref. [16], it has been shown that the combination of electromagnetic and Gross-Neveu interactions yields $\lambda_{c}^{\max }=0.66$, which is our case $(\mathrm{C} 1 \mathrm{~A})$. We believe that combinations with a minimal critical coupling constant $\lambda_{c}^{\min }=0.26$ (see Fig. 4) are likely to provide an easier controlling of the renormalized mass. Indeed, because of screening effects, due to the substrates, the value of $\lambda$ decreases; hence, the phase when $\lambda>\lambda_{c}$ becomes harder to achieve experimentally. From the experimental point of view, it is possible to relate the energy scale $\Lambda$ with the electronic density $n$ (the number of electrons by unit of surface area) by using the scaling law $\Lambda \rightarrow n^{1 / 2}$ [25]. The value of $n$ is controlled by a gate voltage [16]. We believe that our results may be relevant for describing a more realistic process of mass renormalization. Obviously, the four-fermion interactions should be related with microscopic interactions, such as mechanical vibrations, impurities, and disorder in the honeycomb lattice.

\section{SUMMARY AND OUTLOOK}

The experimental realization of two-dimensional materials, where the quasiparticles obey a Dirac-like equation, allow us to consider a quantum-electrodynamical approach in order to describe electronic interactions in these systems. In particular, the experimental observation [26] of the Fermi velocity renormalization [19] in graphene confirms that electronic interactions are indeed relevant. Recently, the description of the band gap renormalization [16] in $\mathrm{WSe}_{2}$ [17] and $\mathrm{MoS}_{2}$ [18] increases this window of possible applications, using standard renormalization group equations, as in Ref. [27]. Within a nonperturbative regime, one can also consider the description of excitonic spectrum [28], dynamical mass generation [29], and the realization of parity anomaly [30] through a quantum valley Hall effect. Beyond these regimes, one can consider the microscopic interactions by taking models that simultaneously describe both electromagnetic and four-fermion interactions. These cases, however, have been less discussed in literature [31].

In this work, we gave a step forward in this picture by considering an effective low-energy model that is suitable 
for calculating the effects of both electromagnetic and the generalized four-fermion interactions with $O(4)$ symmetry. As a concrete application, we calculated the renormalized mass $m(\Lambda)$ within the large- $N$ approximation. This may be measured by looking at the energy gap between the valence (negative energy) and conduction (positive energy) bands at the valleys of the honeycomb lattice $[17,18]$. For the sake of comparison with the experimental data, we may replace the energy scale $\Lambda$ by the electron density $n$, through the transform $\Lambda \rightarrow n^{1 / 2}$, which is true for two-dimensional electrons $[16,19]$. Our result shows that an ultraviolet fixed point $\lambda_{c}$ is generated, implying that $m(\Lambda)$ does not renormalize at $\lambda=\lambda_{c}$. Thereafter, we find that there exist two possible values for $\lambda_{c}$, namely the maximal value $\lambda_{c}^{\max }=$ 0.66 and the minimal value $\lambda_{c}^{\text {min }}=0.26$ (this does not depends on the constant $N$ ). The kind of value we find depends on the combinations of four-fermion interactions we are considering in the initial model. This provides a possible tuning mechanism for the renormalized mass, because the behavior of $m(\Lambda)$ changes when $\lambda=\pi \alpha / 4$ is either larger or less than $\lambda_{c}$.

The model presented here is also suitable for investigating the ultrarelativistic limit of Dirac-like materials, where $v_{F}(n) \rightarrow c$ as $n \rightarrow 0$, where $c$ is the light velocity. Because our current results only describe the regime where $v_{F}(n) \ll c$ (the static limit), it would be interesting to understand the behavior of the renormalized mass in the dynamical limit. We shall consider this generalization elsewhere.

\section{ACKNOWLEDGMENTS}

L.F. is partially supported by Coordenação de Aperfeiçoamento de Pessoal de Nível Superior Brasil (CAPES), finance code 001. V. S. A. and L. O. N. are partially supported by Conselho Nacional de Desenvolvimento Científico e Tecnológico (CNPq) and by CAPES/NUFFIC, finance code 0112. F. P. acknowledges the financial support from Dirección de Investigación de La Universidad de La Frontera Grant No. DI20-0005.

\section{APPENDIX: VACUUM POLARIZATION TENSOR OF FOUR-FERMION INTERACTIONS}

Equation (18) represents the general form of the oneloop quantum correction to the auxiliary-field propagators. Here, we provide a few details of the computation of this term for the case of the Thirring interaction where the vertex is $\varphi_{\mu} \bar{\psi} \gamma^{\mu} \psi$. In this case, we have

$\Pi_{\varphi_{\mu}}^{\mu \nu}(\bar{p})=-\frac{1}{N v_{F}^{2}} \operatorname{Tr} \int \frac{d^{3} k}{(2 \pi)^{3}} \gamma^{\mu} S_{F}(\bar{p}+k) \gamma^{\nu} S_{F}(k)$.

Next, we use Eq. (6) and the following trace operations over the Dirac matrices, namely

$$
\begin{gathered}
\operatorname{Tr}\left[\gamma^{\mu} \gamma^{\nu}\right]=-4 \delta^{\mu \nu}, \\
\operatorname{Tr}\left[\gamma^{\mu} \gamma^{\alpha} \gamma^{\nu} \gamma^{\beta}\right]=4\left(\delta^{\mu \alpha} \delta^{\nu \beta}-\delta^{\mu \nu} \delta^{\alpha \beta}+\delta^{\mu \beta} \delta^{\nu \alpha}\right),
\end{gathered}
$$

which are useful properties to expand the numerator of Eq. (A1). After calculating the trace over the Dirac matrices, this numerator reads

$(\bar{p}+k)^{\mu} k^{\nu}+(\bar{p}+k)^{\nu} k^{\mu}-\delta^{\mu \nu}\left[(\bar{p}+k) \cdot k+m^{2}\right]$.

On the other hand, we use the Feynman parametrization in the denominator, which becomes equal to

$$
\left[(k+x \bar{p})^{2}+x(1+x) \bar{p}^{2}+m^{2}\right]^{2} .
$$

Thereafter, in order to eliminate symmetric-loop integrals, we made a variable change $k \rightarrow k-x \bar{p}$ and, by using Lorentz invariance, we finally find a simplified equation for $\Pi_{\varphi_{\mu}}^{\mu \nu}(\bar{p})$, namely

$$
\begin{aligned}
\Pi_{\varphi_{\mu}}^{\mu \nu}(\bar{p})= & -\frac{4}{v_{F}^{2}} \int_{0}^{1} d x\left\{\int \frac{d^{3} k}{(2 \pi)^{3}} \frac{\left(\frac{2}{3}-1\right) \delta^{\mu \nu} k^{2}}{\left[k^{2}+\Delta_{1}\right]^{2}}\right. \\
& \left.\times \int \frac{d^{3} k}{(2 \pi)^{3}} \frac{\left(\delta^{\mu \nu}-2 \frac{\bar{p}^{\mu} \bar{p}^{\nu}}{\bar{p}^{2}}\right) x(1-x) \bar{p}^{2}-\delta^{\mu \nu} m^{2}}{\left[k^{2}+\Delta_{1}\right]^{2}}\right\},
\end{aligned}
$$

with $\Delta_{1}=x(1-x) \bar{p}^{2}+m^{2}$. After solving the integrals, using the dimensional regularization scheme, we find

$$
\begin{aligned}
\Pi_{\varphi_{\mu}}^{\mu \nu}(\bar{p})= & -\frac{\bar{p}^{2}}{\pi v_{F}^{2}}\left\{\frac{\sqrt{m^{2}}}{2 \bar{p}^{2}}+\frac{\bar{p}^{2}-4 m^{2}}{4 \bar{p}^{2} \sqrt{\bar{p}^{2}}}\right. \\
& \left.\times \arcsin \left[\sqrt{\frac{\bar{p}^{2}}{\bar{p}^{2}+4 m^{2}}}\right]\right\} \overline{\mathbb{P}}^{\mu \nu} .
\end{aligned}
$$

In the cases of other auxiliary fields, we use that $\gamma^{3}$ and $\gamma^{5}$ anticommute with $\gamma^{\mu}$ and between them; furthermore, $\left(\gamma^{3}\right)^{2}=\left(\gamma^{5}\right)^{2}=1$. Hence, it follows some useful properties, given by

$$
\begin{gathered}
\operatorname{Tr}\left[\gamma^{3(5)} \gamma^{3(5)}\right]=4, \\
\operatorname{Tr}\left[\gamma^{3(5)} \gamma^{\alpha} \gamma^{3(5)} \gamma^{\beta}\right]=4 \delta^{\alpha \beta}, \\
\operatorname{Tr}\left[\gamma^{3} \gamma^{5} \gamma^{3} \gamma^{5}\right]=-4, \\
\operatorname{Tr}\left[\gamma^{\mu} \gamma^{3(5)} \gamma^{\alpha} \gamma^{\nu} \gamma^{3(5)} \gamma^{\beta}\right]=\operatorname{Tr}\left[\gamma^{\mu} \gamma^{\alpha} \gamma^{\nu} \gamma^{\beta}\right], \\
\operatorname{Tr}\left[\gamma^{3} \gamma^{5} \gamma^{\alpha} \gamma^{3} \gamma^{5} \gamma^{\beta}\right]=4 \delta^{\alpha \beta} \\
\operatorname{Tr}\left[\gamma^{\mu} \gamma^{3} \gamma^{5} \gamma^{\alpha} \gamma^{\nu} \gamma^{3} \gamma^{5} \gamma^{\beta}\right]=-\operatorname{Tr}\left[\gamma^{\mu} \gamma^{\alpha} \gamma^{\nu} \gamma^{\beta}\right] .
\end{gathered}
$$


We obtain $\Pi_{\varphi_{3}}(\bar{p})$ and $\Pi_{\varphi_{5}}(\bar{p})$ using $\Gamma^{l}=\gamma^{3}$ or $\gamma^{5}$, respectively, in Eq. (18). Then, we implement the same procedure for solve Eq. (A1) together with Eqs. (A8) and (A9), of form that

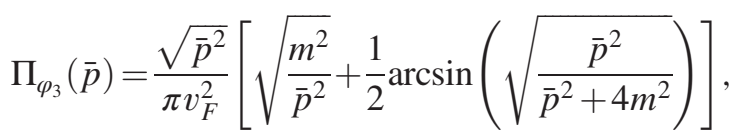

and for $\Pi_{\varphi_{5}}(\bar{p})$ we have the same previous result. Using $\Gamma^{l}=\gamma^{\mu} \gamma^{3}$ (or $\left.\gamma^{\mu} \gamma^{5}\right)$ in Eq. (18) and Eqs. (A9) and (A11), we find $\Pi_{\varphi_{\mu 3}}(\bar{p})\left[\right.$ or $\left.\Pi_{\varphi_{\mu 5}}(\bar{p})\right]$, namely

$$
\begin{aligned}
\Pi_{\varphi_{\mu 3}}^{\mu \nu}(\bar{p})= & -\frac{\sqrt{\bar{p}^{2}}}{2 \pi v_{F}^{2}}\left\{\left[\sqrt{\frac{m^{2}}{\bar{p}^{2}}+\frac{\bar{p}^{2}+4 m^{2}}{2 \bar{p}^{2}}}\right.\right. \\
& \left.\left.\times \arcsin \left(\sqrt{\frac{\bar{p}^{2}}{\bar{p}^{2}+4 m^{2}}}\right)\right] \overline{\mathbb{P}}^{\mu \nu}+2 \frac{m^{2}}{\bar{p}^{2}} \delta^{\mu \nu}\right\} ;
\end{aligned}
$$

using $\Gamma^{l}=\gamma^{3} \gamma^{5}$ in Eq. (18) together with Eqs. (A10) and (A12), we obtain

$$
\begin{aligned}
\Pi_{35}(\bar{p})= & -\frac{\sqrt{\bar{p}^{2}}}{\pi v_{F}^{2}}\left[\sqrt{\frac{m^{2}}{\bar{p}^{2}}+\frac{\bar{p}^{2}+4 m^{2}}{2 \bar{p}^{2}}}\right. \\
& \left.\times \arcsin \left(\sqrt{\frac{\bar{p}^{2}}{\bar{p}^{2}+4 m^{2}}}\right)\right],
\end{aligned}
$$

and last, we may obtain $\Pi_{\varphi_{\mu 35}}^{\mu \nu}(\bar{p})$ by replacing $\Gamma^{l}$ by $\gamma^{\mu} \gamma^{3} \gamma^{5}$ in Eq. (18) and using the trace operation given by Eqs. (A12) and (A13), so

$$
\begin{aligned}
\Pi_{\varphi_{\mu 35}}^{\mu \nu}(\bar{p})= & \frac{\bar{p}^{2}}{\pi v_{F}^{2}}\left[\frac{\sqrt{m^{2}}}{2 \bar{p}^{2}}+\frac{\bar{p}^{2}-4 m^{2}}{4 \bar{p}^{2} \sqrt{\bar{p}^{2}}}\right. \\
& \left.\times \arcsin \left(\sqrt{\frac{\bar{p}^{2}}{\bar{p}^{2}+4 m^{2}}}\right)\right] \overline{\mathbb{P}}^{\mu \nu} .
\end{aligned}
$$

[1] W. Thirring, A soluble relativistic field theory, Ann. Phys. (N.Y.) 3, 91 (1958).

[2] Y. Nambu and G. Jona-Lasinio, Dynamical model of elementary particles based on an analogy with superconductivity. I, Phys. Rev. 122, 345 (1961); Dynamical model of elementary particles based on an analogy with superconductivity. II, Phys. Rev. 124, 246 (1961).

[3] R. T. Cahill and C. D. Roberts, Soliton bag models of hadrons from QCD, Phys. Rev. D 32, 2419 (1985); D. Dyakonov and V. Yu Petrov, A theory of light quarks in the instanton vacuum, Nucl. Phys. B272, 457 (1986); H. Reinhardt, Hadronization of quark flavor dynamics, Phys. Lett. B 244, 316 (1990); Bosonization of QCD in the field strength approach, Phys. Lett. B 257, 375 (1991).

[4] T. Hatsuda and T. Kunihiro, QCD phenomenology based on a chiral effective Lagrangian, Phys. Rep. 247, 221 (1994).

[5] F. J. Dyson, The radiation theories of tomonaga, Schwinger, and Feynman, Phys. Rev. 75, 486 (1949); The $S$ matrix in quantum electrodynamics, Phys. Rev. 75, 1736 (1949).

[6] C. de Calan, P. A. Faria da Veiga, J. Magnen, and R. Sénéor, Constructing the Three-Dimensional Gross-Neveu Model with a Large Number of Flavor Components, Phys. Rev. Lett. 66, 3233 (1991); H. Gies and L. Janssen, UV fixedpoint structure of the three-dimensional Thirring model, Phys. Rev. D 82, 085018 (2010).

[7] D. J. Gross, in Proceedings of Lês Houches, Session XXVIII, 1975, edited by R. Balian and J. Zinn-Justin (North-Holland Publishing Co, Amsterdam, 1976); B. Rosenstein, B. J. Warr, and S. H. Park, Four-Fermion Theory is Renormalizable in $2+1$ Dimensions, Phys. Rev. Lett. 62, 1433 (1989).
[8] M. Gomes, V. O. Rivelles, and A. J. da Silva, Dynamical parity violation and the Chern-Simons term, Phys. Rev. D 41, 1363 (1990); M. Gomes, R. S. Mendes, R. F. Ribeiro, and A. J. da Silva, Gauge structure, anomalies, and mass generation in a three-dimensional Thirring model, Phys. Rev. D 43, 3516 (1991).

[9] J. Hubbard, Calculation of Partition Functions, Phys. Rev. Lett. 3, 77 (1959).

[10] K. S. Novoselov, A. K. Geim, S. V. Morozov, D. Jiang, Y. Zhang, S. V. Dubonos, I. V. Grigorieva, and A. A. Firsov, Electric field effect in atomically thin carbon films, Science 306, 666 (2004).

[11] B. Lalmi, H. Oughaddou, H. Enriquez, A. Kara, S. Vizzini, B. Ealet, and B. Aufray, Epitaxial growth of a silicene sheet, Appl. Phys. Lett. 97, 223109 (2010).

[12] G. Wang, A. Chernikov, M. M. Glazov, T. F. Heinz, X. Marie, T. Amand, and B. Urbaszek, Colloquium: Excitons in atomically thin transition metal dichalcogenides, Rev. Mod. Phys. 90, 021001 (2018).

[13] P.-L. Zhao, A.-M. Wang, and G.-Z. Liu, Condition for the emergence of a bulk Fermi arc in disordered Dirac-fermion systems, Phys. Rev. B 98, 085150 (2018).

[14] J. Wang, Role of four-fermion interaction and impurity in the states of two-dimensional semi-Dirac materials, J. Phys. Condens. Matter 30, 12 (2018).

[15] E. C. Marino, Quantum electrodynamics of particles on a plane and the Chern-Simons theory, Nucl. Phys. B408, 551 (1993).

[16] L. Fernández, V.S. Alves, L. O. Nascimento, F. Penña, M. Gomes, and E. C. Marino, Renormalization of the band gap in $2 \mathrm{D}$ materials through the competition between 
electromagnetic and four-fermion interactions in large $\mathrm{N}$ expansion, Phys. Rev. D 102, 016020 (2020).

[17] P. V. Nguyen, N. C. Teutsch, N. P. Wilson, J. Kahn, X. Xia, A. J. Graham, V. Kandyba, A. Giampietri, A. Barinov, G. C. Constantinescu, N. Yeung, N. D. M. Hine, X. Xu, D. H. Cobden, and N. R. Wilson, Visualizing electrostatic gating effects in two-dimensional heterostructures, Nature (London) 572, 220 (2019).

[18] F. Liu, M. E. Ziffer, K. R. Hansen, J. Wang, and X. Zhu, Direct Determination of Band-Gap Renormalization in the Photoexcited Monolayer $\mathrm{MoS}_{2}$, Phys. Rev. Lett. 122, 246803 (2019).

[19] M. A. H. Vozmediano and F. Guinea, Effect of Coulomb interactions on the physical observables of graphene, Phys. Scr. T146, 014015 (2012); F. de Juan, A. G. Grushin, and M. A. H. Vozmediano, Renormalization of Coulomb interaction in graphene: Determining observable quantities, Phys. Rev. B 82, 125409 (2010); J. González, F. Guinea, and M. A. H. Vozmediano, Marginal-Fermi-liquid behavior from two-dimensional Coulomb interaction, Phys. Rev. B 59, R2474 (1999).

[20] B. Charneski, M. Gomes, T. Mariz, J. R. Nascimento, and A. J. da Silva, Dynamical Lorentz symmetry breaking in 3D and charge fractionalization, Phys. Rev. D 79, 065007 (2009).

[21] N. Menezes, V. S. Alves, E. C. Marino, L. Nascimento, L. O. Nascimento, and C. M. Smith, Spin $g$-factor due to electronic interactions in graphene, Phys. Rev. B 95, 245138 (2017).

[22] E. C. Marino, Quantum Field Theory Approach to Condensed Matter Physics (Cambridge University Press, Cambridge, England, 2017).
[23] J.-R. Wang, G.-Z. Liu, and C.-J. Zhang, Renormalization of fermion velocity in finite temperature $\mathrm{QED}_{3}$, Phys. Rev. D 93, 045017 (2016).

[24] T. Son, Quantum critical point in graphene approached in the limit of infinitely strong Coulomb interaction, Phys. Rev. B 75, 235423 (2007).

[25] S. D. Sarma, S. Adam, E. H. Hwang, and E. Rossi, Electronic transport in two-dimensional graphene, Rev. Mod. Phys. 83, 407 (2011); S. D. Sarma, E. H. Hwang, and H. Min, Carrier screening, transport, and relaxation in three-dimensional Dirac semimetals, Phys. Rev. B 91, 035201 (2015).

[26] D. C. Elias, R. V. Gorbachev, A. S. Mayorov, S. V. Morozov, A. A. Zhukov, P. Blake, L. A. Ponomarenko, I. V. Grigorieva, K. S. Novoselov, F. Guinea, and A. K. Geim, Dirac cones reshaped by interaction effects in suspended graphene, Nat. Phys. 7, 701 (2011).

[27] M. S. Foster and I. L. Aleiner, Grapehen via large N: A renormalization group study, Phys. Rev. B 77, 195413 (2008).

[28] E. C. Marino, L. O. Nascimento, V. S. Alves, N. Menezes, and C.M. Smith, Quantum-electrodynamical approach to the exciton spectrum in transition-metal dichalcogenides, 2D Mater. 5, 041006 (2018).

[29] C. Popovici, C. S. Fischer, and L. von Smekal, Fermi velocity renormalization and dynamical gap generation in graphene, Phys. Rev. B 88, 205429 (2013).

[30] E. C. Marino, L. O. Nascimento, V.S. Alves, and C. M. Smith, Interaction Induced Quantum Valley Hall Effect in Graphene, Phys. Rev. X 5, 011040 (2015).

[31] D. Ebert, K. G. Klimenko, P. B. Kolmakov, and V. Ch. Zhukovsky, Phase transitions in hexagonal, graphene-like lattice sheets and nanotubes under the influence of external conditions, Ann. Phys. (Amsterdam) 371, 254 (2016). 